\title{
Dermoscopic surprises in a series of 6 cases
}

\section{Subrata Malakar, Purva Mehta, Samipa Mukherjee, Surit Malakar}

\author{
Rita Skin Foundation, Kolkata, India
}

Corresponding author: Dr. Purva Mehta, E-mail: purvamehta86@gmail.com

\begin{abstract}
Dermoscopy is a non invasive technique which can be a fantastic diagnostic tool in dermatology. Here we present a series of six cases where a dermoscopy threw light on the clinical diagnosis of lesions. Case 1A, 1B and IC presented with singular papular lesions which led us to differential diagnosis of viral infections, seborrheic keratoses etc. However, on dermoscopy all three cases exhibited a white mass with a keratin plug and peripheral hair pin blood vessels. Our second case presented with a nodule on the nose leading us to a diagnosis of an intradermal nevus. Dermoscopy of this lesion demonstrated the presence of arborizing vessels thus indicating something far more sinister than a nevus. Case 3 was a female who presented with a small asymptomatic lobulated mass leading us to consider either seborrheic keratosis or dermatofibroma as diagnosis. But the dermoscopy correctly showed the presence of lacunae and a whitish veil which established the final diagnosis. Our final case presented with a greyish nodule suggestive of dermatofibroma. On dermoscopy, the presence of cerebriform structures and comedo like openings on the background of a white scar like patch highlighted the superimposition of another lesion on the existing dermatofibroma. Our cases prove the utility of dermoscopy as a diagnostic tool in clinical dermatology.
\end{abstract}

Key words: Dermoscopy; Hair pin vessels; Arborizing vessels

\section{INTRODUCTION}

Dermoscopy has revolutionised the non invasive methodology of clinical diagnosis in the field of dermatology. It not only exhibits the subsurface features invisible to the naked eye but also guides us to an accurate diagnosis. Here, we present a conundrum of several cases where the discrepancy between our clinical suspicion and the actual diagnosis was highlighted by the simple yet effective use of a dermoscopy.

\section{CASE REPORT}

\section{Case 1A}

A 40 year old male presented to us with a lesion on the upper lip since a few years. The lesion was asymptomatic and static in nature. On cutaneous examination, there was a singular, firm, sessile, skin coloured papular lesion measuring $0.5 \times 0.5 \mathrm{~cm}$ in size, with two to three hair protruding from it (Fig. 1).
On the basis of clinical examination we came to a differential diagnosis of compound nevus, verruca vulgaris, molluscum contagiosum and seborrheic keratosis. On dermoscopy, there was a white mass with central yellow keratin crusts surrounded by a paler dull white structureless zone. Blood spots were also noticeable in the centre of the white keratin mass. At the periphery of the mass there was a radial disribution of hair pin blood vessels (Fig. 2).

Thus the dermoscopic examination clinched the diagnosis of keratoacanthoma.

\section{Case 1B}

A 35 year old female patient presented to us with a lesion on the nose since a couple of years. The lesion was asymptomatic in nature and static in size. Cutaneous examination revealed a single, firm, pea sized, brown to black coloured sessile papule on the nose (Fig. 3). 


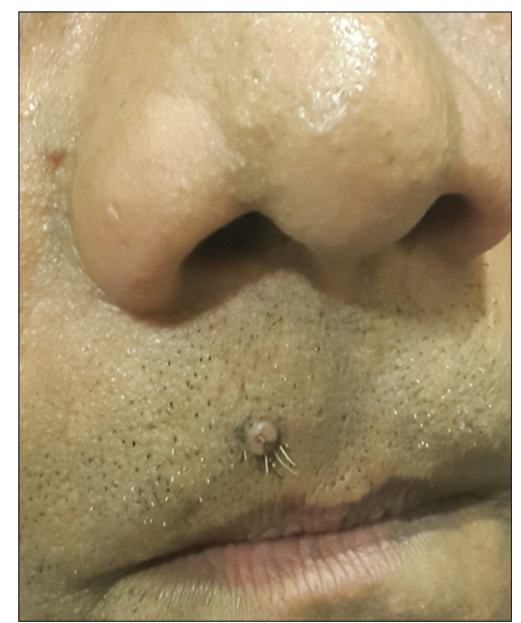

Figure 1: Singular, firm, sessile, skin coloured papular lesion on the upper lip.

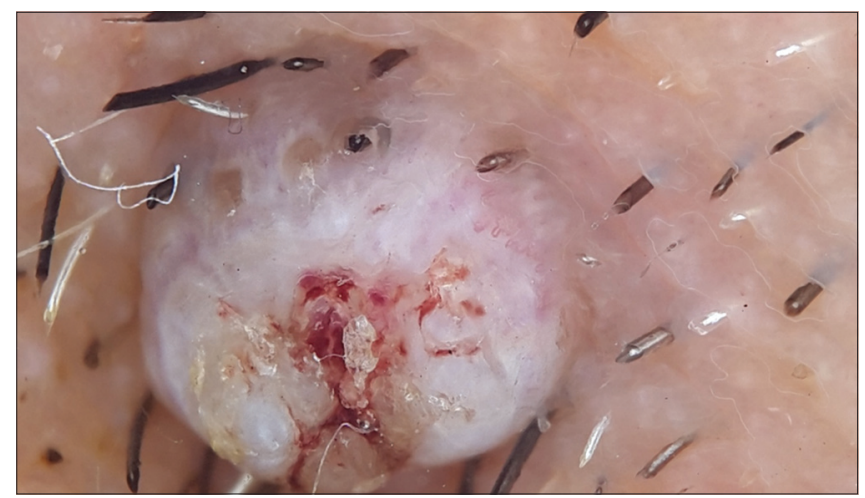

Figure 2: White coloured structure with central yellow keratin crusts and blood spots with hair pin blood vessels (arrow) arranged along the periphery of the structure.

Considering the clinical appearance of the lesion, we thought of seborrheic keratosis, compound nevus, verruca vulgaris and solitary fibrous papule of the nose as differential diagnosis. To confirm our clinical suspicion we used a dermoscopy. On dermoscopic examination, there was a dome shaped white mass. At the centre of this dome shaped mass, there was a keratin plug which was surrounded by a white structureless zone. At the periphery of the mass, we observed hair pin blood vessels (Fig. 4).

This fulfilled most of the dermoscopic diagnostic criteria for keratoacanthoma and a final diagnosis of solitary keratoacanthoma was made.

\section{Case 1C}

A 43 year old male presented with a lesion on his left forearm since few months. The lesion was essentially asymptomatic in nature with no change in size. Cutaneous examination revealed a solitary, skin

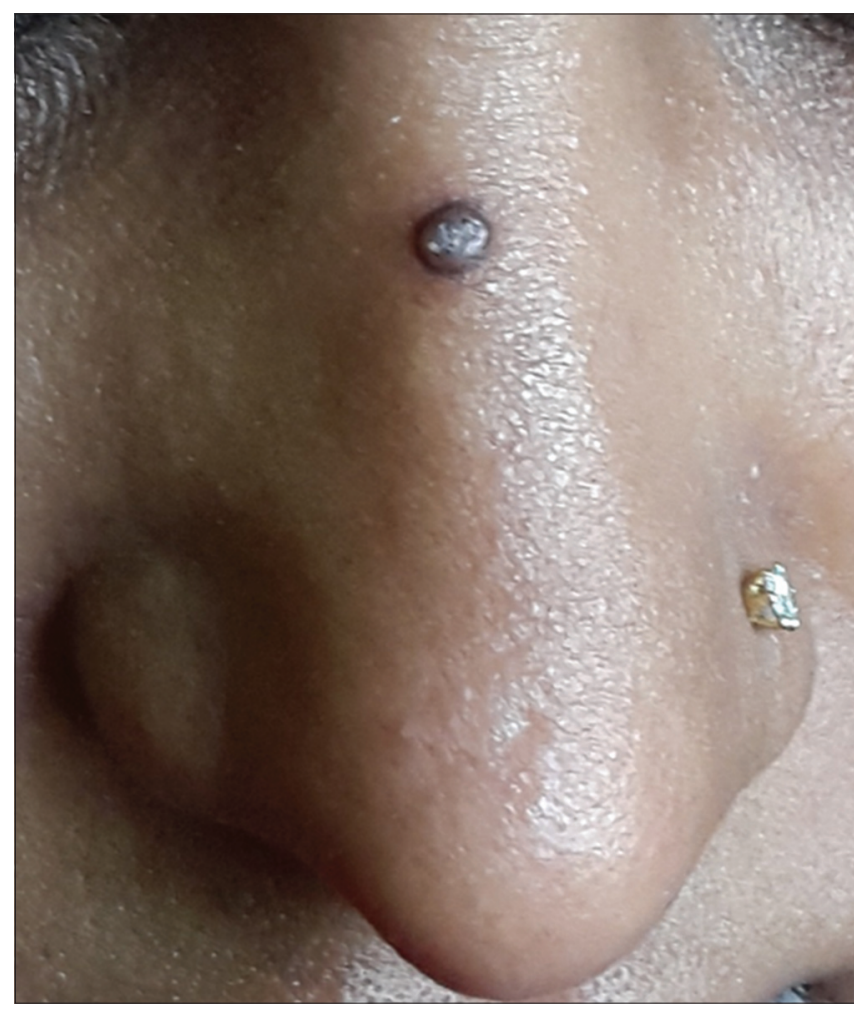

Figure 3: Single, firm, pea sized, brown to black coloured sessile papule on the nose.

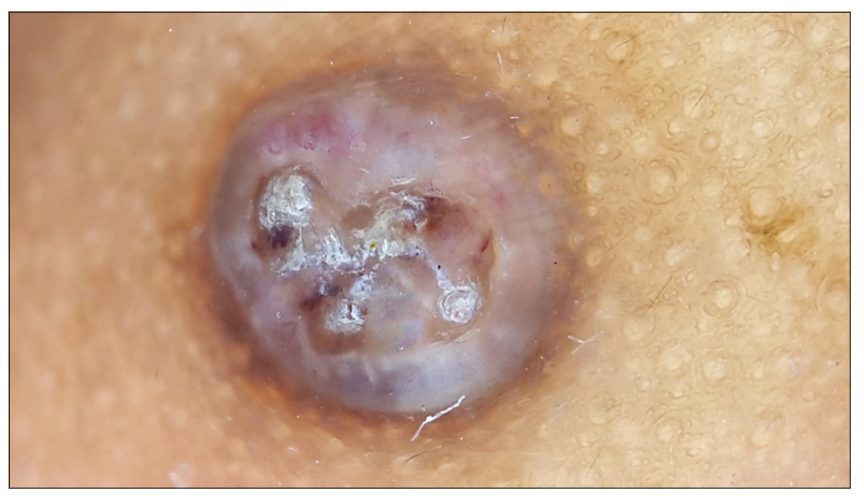

Figure 4: Dome shaped mass with central keratin and peripheral hair pin vessels (arrow).

coloured dome shaped nodule with central scaling (Fig. 5).

Clinical appearance led us to molluscum contagiosum or verruca vulgaris as differential diagnosis. Dermoscopy, however revealed a surprisingly different picture. On dermoscopic examination, there was a white mass with central yellow keratin surrounded by a white structureless zone (Fig. 6).

A dermoscope yet again surprised us as it confirmed that the lesion was a keratoacanthoma and ruled out our clinical suspicions completely. 


\section{Case 2}

A 45 year old male presented to us with a lesion on the nose since a few months. The lesion was asymptomatic in nature but had gradually increased in size over the last one month. On cutaneous examination, there was a solitary, skin coloured, ovoid nodule measuring $1.5 \mathrm{X}$ $1.5 \mathrm{cms}$ on the nose (Fig. 7)

The differentials that came to our mind were intradermal nevus, solitary angiofibroma and solitary fibrous papule of the nose.

However, the non invasive technique of dermoscopy had a surprise in store. Dermoscopy of the nasal lesion revealed arborising telangiectatic vessels coursing throughout the lesion. These vessels were branching at irregular intervals into thin capillaries and were bright red in colour. On closer examination, dermoscopy also highlighted that these arborizing vessels were sharp in focus and their borders were abruptly cut off (Fig. 8).

The dermoscopic picture was diagnostic of basal cell carcinoma. Arborising vessels are not a dermoscopic feature of either nevus, angiofibroma or solitary fibrous papule of nose. Dermoscopy thus eliminated all our clinical suspicions and led us to an accurate diagnosis. The patient was then subjected to an excision biopsy and the excised specimen was sent for histopathological examination.

Histological analysis showed nests of basaloid epithelial cells in the dermis with hyperchromatic nucelii and a peripheral palisading pattern all embedded in fibrous septae. Keratin pearls were also present in the dermis. Thus, histopathology confirmed our dermoscopic findings and labelled the lesion to be keratotic basal cell carcinoma (Fig. 9).

\section{Case 3}

A 22 year old female presented to us with a lesion on the right knee since several years. The lesion was essentially asymptomatic and stable in size. Cutaneous examination revealed a singular, gray to brown coloured, lobulated mass measuring $1 \mathrm{~cm} \mathrm{X} 0.5 \mathrm{~cm}$ in size on the right knee (Fig. 10).

Clinical appearance of the lesion led us to a differential diagnosis of seborrheic keratosis and dermatofibroma. However, the use of dermoscopy, yet again proved

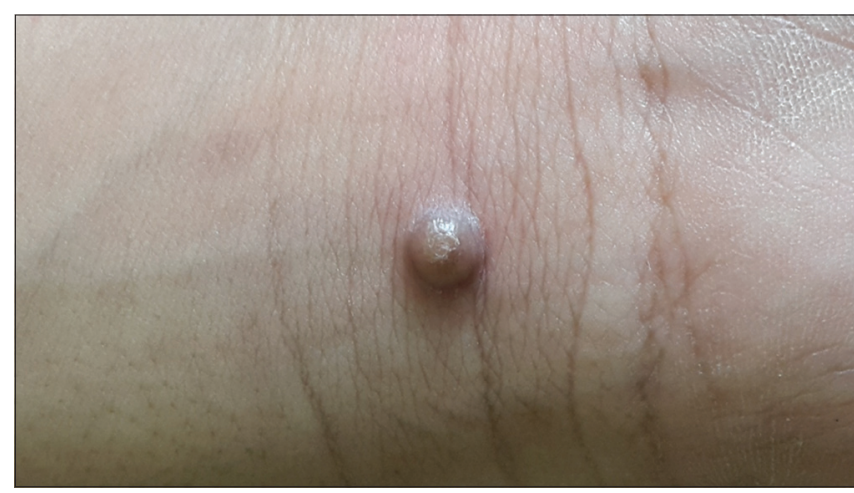

Figure 5: Solitary, skin coloured dome shaped nodule with centra scaling.

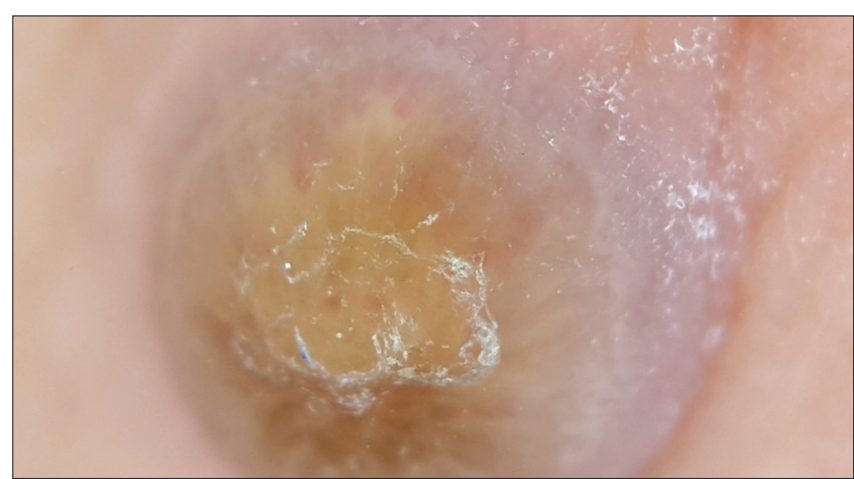

Figure 6: White coloured mass with central yellow keratin (arrow) surrounded by a white structureless zone.

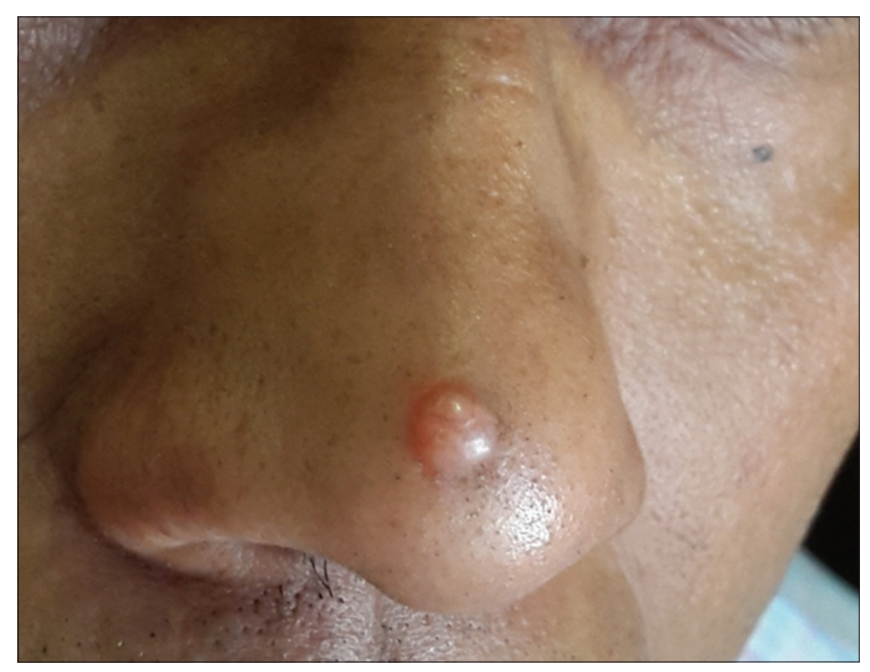

Figure 7: Solitary, skin coloured, ovoid papulonodule on the nose.

valuable in unmasking the accurate diagnosis. On dermoscopy, there were several, round to oval well demarcated red to blue lacunae with a whitish veil (Fig. 11).

The dermoscopic feature was diagnostic of angiokeratoma ruling out all our clinical suspicions. 


\section{Case 4}

A 57 year old female came with complaints of a lesion on the left thigh which was present since several years. She had no symptoms regarding the lesion and it was static in size. On clinical examination, there was a violaceous to grey nodule measuring $1 \times 0.5 \mathrm{cms}$ in size which was firm in consistency (Fig. 12).

The clinical appearance of the lesion was suggestive of dermatofibroma. However, the dermoscopy as always had more to convey. Dermoscopic examination revealed cerebriform structures with comedo like openings on the background of a white scar like patch (Fig. 13).

\section{DISCUSSION}

Epiluminescence microscopy in these 3 cases was an eye opener as the clinical picture and the actual diagnosis were miles apart. The white mass with central blood spots and keratin crusts along with the characteristic peripheral arrangement of hair pin blood vessels is diagnostic of keratoacanthoma [1]. Also, the peripheral arrangement of hair pin blood vessels favours a diagnosis of keratoacanthoma over seborrheic keratosis in which the hair pin vessels are evenly distributed throughout the lesion [2]. The use of dermoscopy ruled out one of our clinical suspicions of compound nevus as comma vessels; which are a key dermoscopic feature of intradermal melanocytic nevi were missing [3]. The dermoscopic vascular pattern of homogenous dots and globules, red to black in colour was absent ruling out verruca vulgaris [4]. The absence of crown vessels at the periphery in a radial distribution ruled out another clinical differential diagnosis of molluscum contagiosum [5].

The presence of hair around the lesion in case 1A was intriguing. That could be attributed to the hesitancy of the patient to shave or trim that particular area owing to the presence of the lesion.

Keratoacanthoma and squamous cell carcinoma are quite indistinguishable clinically and dermoscopically. However, the presence of central keratin favours a diagnosis of keratoacanthoma over squamous cell carcinoma. This finding is crucial as it correlates to a central keratin plug on histopathology of keratoacanthoma [1].

Dermoscopy is an excellent non invasive tool as it not only maginifies the subtle characteristics of a lesion but also throws light on the vascular patterns of the lesion. Identification of the vascular pattern is often imperative for the diagnosis of basal cell carcinoma as most of them are non pigmented. Arborising branching vessels which are hallmark for basal cell carcinoma are defined as large calibre vessels which are in focus and branch into finer secondary vessels. Arborising branching vessels are a dermoscopic feature of both basal cell carcinoma and adnexal tumours. However the clinical picture went against the diagnosis of an adnexal tumour as the lesion was solitary and larger in size whereas adnexal tumours tend to be multiple and smaller in size. The dermoscopy also helps to pinpoint the location of the

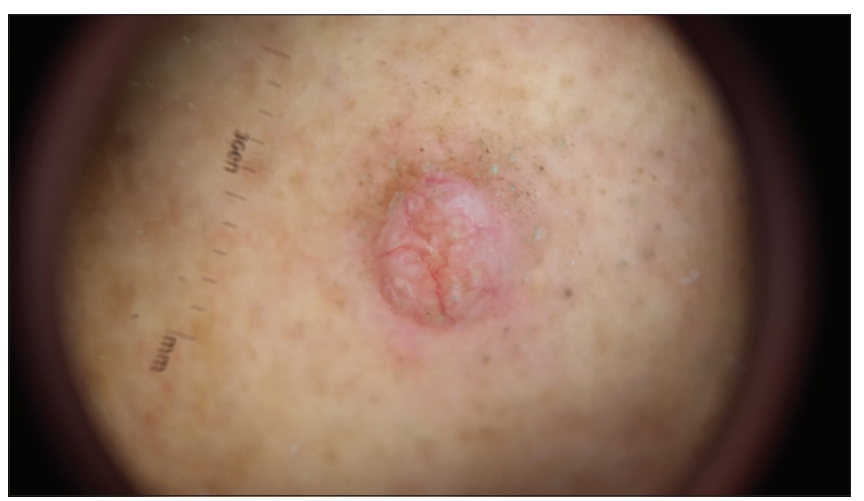

Figure 8: Arborising telangiectatic vessels (arrow) coursing throughout the lesion.

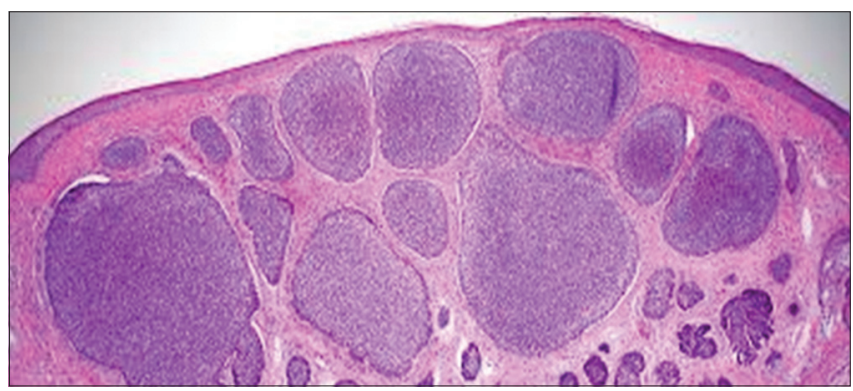

Figure 9: Nests of basaloid cells in the dermis with hyperchromatic nuclei and a peripheral palisading pattern.

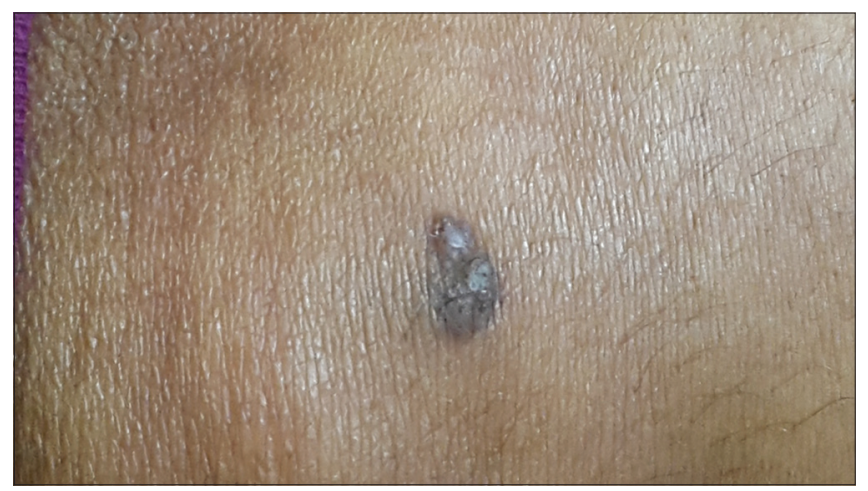

Figure 10: Singular, soft to firm, grey brown lobulated mass on the right knee. 
blood vessels by highlighting the colour of the vessels and their characteristic morphology and architectural arrangement. The linear pattern of the blood vessels in our case confirmed their superficial location in the dermis as deeper dermal vessels would have appeared as pin point dots. The linearity of the blood vessels also suggested that they ran parallel to the skin surface where as blood vessels that ran perpendicular to the skin surface would have been viewed as dots or loops. Also the vessels were bright red in colour, prominent to the eye and "in focus" reaffirming their location in the upper dermis. On the other hand, connective tissue in the dermis causes dispersion of light and this would make deeper dermal vessels appear as pink dots, less prominent and out of focus [2].

Dermoscopy once again confirmed that clinical appearances are often misleading and appreciation of subsurface features can lead us to a correct diagnosis. The presence of red to blue lacunae with a whitish veil are diagnostic of angiokeratomas on dermoscopy. In this case, there was absence of hair pin vessels which are a diagnostic hallmark for seborrheic keratosis. The absence of dotted, comma or hairpin vessels ruled out our clinical suspicion of dermatofibroma [6]. Also the diagnostic dermoscopic features of dermatofibroma like pigment network and central white patch were missing [7]. The lacunae in angiokeratomas histologically correspond to dilated vessels which are either partially or completed thrombosed in the dermis. Hyperkeratosis and acanthosis are the histological markers for the whitish veil found on dermoscopy [8].

Dermoscopy thus revealed that in addition to our clinical diagnosis of dermatofibroma, there was a lesion of seborrheic keratosis which was superimposed on top of the dermatofibroma. There are several patterns of dermatofiroma on dermoscopy. Out of all the patterns, peripheral delicate pigment like network with central white scar like patch is the most common. However, in some cases, dermatofibromas may exhibit only a white scar like patch with no pigment network as in our case. The white scar like patch is a sharply demarcated but irregularly defined white area. It histologically correlates to fibrosis in the papillary dermis [9]. Comedo like openings and milia like cysts are the classical diagnostic criteria for seborrheic keratosis on dermoscopy. However, apart from the classical criteria, presence of other criteria like cerebriform structures or brain like appearance improves the diagnostic accuracy of seborrheic keratoses. The presence of multiple fissures contribute

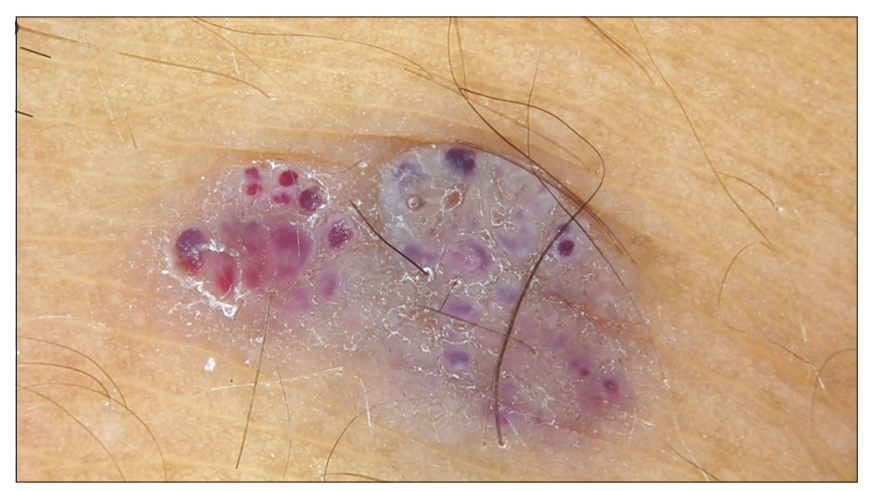

Figure 11: Well demarcated, round to oval, red to blue lacunae (arrow) with a whitish veil.

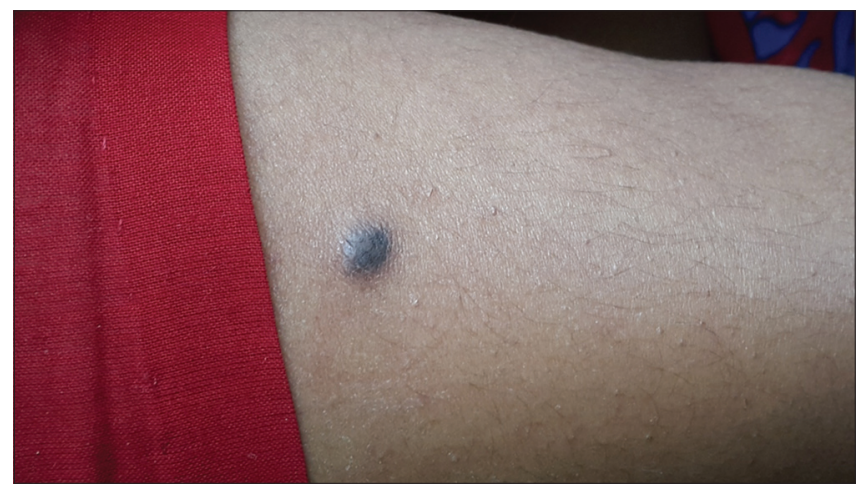

Figure 12: Single, violaceous to grey, firm nodule on the left thigh.

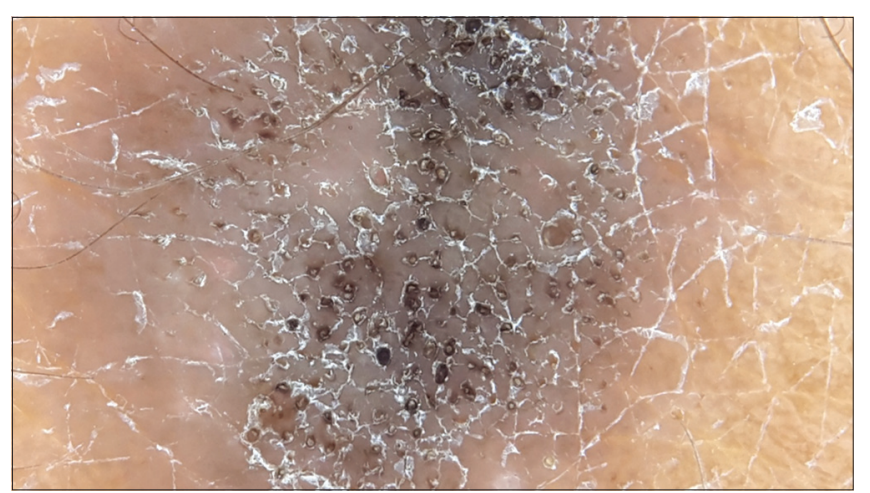

Figure 13: Cerebriform structures (red arrow) with comedo like openings (black arrow) against a background of scar like white patch (blue arrow).

\section{CONCLUSION}

We cannot further emphasize the importance of dermoscopy in clinical practice. In all our cases, dermoscopy took us by surprise as the dermoscopic diagnosis was vastly different than what met the eye. In each case, dermoscopy revealed surprising findings underlining the fact that dermatological lesions have heterogenous and atypical appearances and clinical acumen alone may not be sufficient. In such cases, the simple use of a non invasive technique like dermoscopy 
can do wonders to lead us to an accurate and informed diagnosis. We beseech the dermatologiocal community to use a dermoscopy as a regular out patient tool as the outcome is often astonishing.

\section{REFERENCES}

1. Rosendahl C, Cameron A, Argenziano G, Zalaudek I, Tschandl P, Kittler H. Dermoscopy of Squamous Cell Carcinoma and Keratoacanthoma. Arch Dermatol. 2012;148:1-7.

2. Zalaudek I, Kreusch J, Giacomel J, Ferrara G, Catricalà C, Argenziano G. How to diagnose non pigmented skin tumours: A review of vascular structures seen with dermoscopy. Part I. Melanocytic skin tumours. J Am Acad Dermatol. 2010;63:361-74.

3. Argenziano G, Zalaudek I, Corona R, Sera F, Cicale L, Petrillo G, et al. Vascular structures in skin tumours. A dermoscopic study. Arch Dermatol. 2004;140:1485-19.

4. Bae JM, Kang H, Kim HO. Differential diagnosis of plantar wart from corn, callus and healed wart with the aid of dermoscopy. $\mathrm{Br}$ J Dermatol. 2009;160:220-2.

5. Zalaudek I, Kreusch J, Giacomel J, Ferrara G, Catricalà C,
Argenziano G. How to diagnose non pigmented skin tumours: A review of vascular structures seen with dermoscopy. Part II. Non melanocytic skin tumours. J Am Acad Dermatol. 2010;63:377-86.

6. Martin JM, Bella-Navarro R, Jorda E. Vascular patterns in Dermoscopy. Actas Dermosifiliogr. 2012;103:357-75.

7. Arpaia N, Cassano N, Vena GA. Dermoscopic patterns of dermatofibroma. Dermatol Surg. 2005;31:1336-9.

8. Zaballos P, Daufi C, Puig S, Argenziano G, Moreno-Ramirez D, Cabo H, et al. Dermoscopy of solitary angiokeratoma: a morphological study. Arch Dermatol. 2007;143:318-25.

9. Zaballos P, Puig S, Llambrich A, Malvehy J. Dermoscopy of Dermatofibromas: A Prospective Morphological Study of 412 Cases. Arch Dermatol. 2008;144:75-83.

10. Braun RP, Rabinovitz HS, Krischer J. Dermoscopy of Pigmented Seborrheic Keratosis: A Morphological Study. Arch Dermatol. 2002;138:1556-60.

Copyright by Subrata Malakar, et al. This is an open-access article distributed under the terms of the Creative Commons Attribution License, which permits unrestricted use, distribution, and reproduction in any medium, provided the original author and source are credited.

Source of Support: Nil, Conflict of Interest: None declared. 